\title{
GENERATION OF ABSTRACT-PARAMETER SEMIGROUPS
}

\author{
V. A. BABALOLA ${ }^{1}$
}

ABSTRACT. This paper generalizes the Hille-Yosida-Phillips theorem for abstract-parameter semigroups.

1. Introduction. Generation is a fundamental problem in one-parameter operator semigroup theory and it has been done by many authors. (See, for instance, [3], [1] and the references they contain.) It is, in fact, introducing and characterising an operator, the infinitesimal generator, which determines the structure of the semigroup. In 1944, E. Hille introduced a study of operator semigroups with abstract parameter [3, pp. 327-336 and Chapter XXV]. This has since been studied by Tulcea [8], Langlands [5], [6], Ivanova [4], Chabrowski and Lubzonok [2] and others. However, the notion of the infinitesimal generator for abstract-parameter semigroups, in general, does not seem feasible making it difficult to discuss its generation as above. The main purpose of this paper is to discuss the generation of this kind of semigroup under a suitable generalized notion of generation. Specifically, it involves replacing the "infinitesimal generator" naturally with the cone of infinitesimal generators associated with an abstract-parameter semigroup and characterising cones which give rise to such semigroups. This, indeed, is a generalization of the notion of generation above, since for the one-parameter case characterising the infinitesimal generator or the cone of infinitesimal generators is equivalent.

2. Preliminaries. Let $X, Y$ be complex or real $B$-spaces. Let $X^{+}$be an open positive cone in $X$ (that is, $X^{+}$is an open subset of $X$ such that if $x$, $x^{\prime} \in X^{+}$and $\alpha>0$, then $x+x^{\prime} \in X^{+}$and $\left.\alpha x \in X^{+}\right)$. Let $X^{+}$have the origin of $X$ as a limit point. Let $S=\left\{S(x): x \in X^{+}\right\}$be a semigroup of bounded linear operators on $Y$. Thus $S\left(x+x^{\prime}\right) y=S(x) S\left(x^{\prime}\right) y \forall x, x^{\prime} \in X^{+}, y \in Y$. $S$

Received by the editors December 3, 1973. 46B99.

AMS (MOS) subject classifications (1970). Primary 47 A50, 47D05; Secondary

Key words and phrases. Abstract-parameter semigroup generation, (infinitesimal) generators, (partially) $C_{0}$ semigroups, resolvents.

1 This research was supported in part by Naiontal Science Foundation grant GP $36418 \times 1$. 
is called a partially $C_{0}$ semigroup on $Y$ iff $\lim _{t 10} S(t x) y=y$ for each $x \in X^{+}$and $y \in Y$. It is called a (totally) $C_{0}$ semigroup on $Y$ iff $\lim _{\|x\| \rightarrow 0} S(x) y=y$ for each $y \in Y$. It is clear that if $X^{+}$is finite dimensional then a partially $C_{0}$ semigroup is $C_{0}$. However, $C_{0}$ semigroups are partially $C_{0}$.

Since $X^{+}$is locally bounded, a $C_{0}$ semigroup $S$ is strongly continuous on $X^{+}$(cf. [8, pp. 59-60]). Moreover, it still retains the exponential bound well known in the case $X^{+}=(0, \infty)$.

Theorem 1. Let the semigroup $S$ be $C_{0}$. Then there exist positive constants $\sigma$ and $M$ such that

$$
\|S(x)\| \leq M e^{\|x\| \sigma} \quad \forall x \in X^{+} .
$$

Further, if $B \subset X^{+}$is bounded then there exists $\sigma_{B} \geq 0$ such that

$$
\|S(t x)\| \leq M e^{t \sigma_{B}} \quad \forall t>0, x \in B .
$$

Proof. For $\delta>0$, let $N(\delta)=\left\{x \in X^{+}:\|x\| \leq \delta\right\}$. A familiar uniform boundedness argument shows that there are constants $\delta>0$ and $M=M(\delta) \geq$ 1 such that $\|S(x)\| \leq M \forall x \in N(\delta)$. Now there is $\nu_{0}>0$ such that $\nu N(1) \subset$ $N(\delta) \forall 0<\nu \leq \nu_{0}$. Thus $\|S(\nu x)\| \leq M \quad \forall x \in N(1), 0<\nu \leq \nu_{0}$. Now given any $x \in X^{+}$, there is $0<\tau \leq \nu_{0}$ and a nonnegative integer $m$ such that $\|x\|=$ $m \nu_{0}+\tau$. Hence using a modification of a technique of Miyadera,

$$
\begin{aligned}
\|S(x)\| & =\left\|S\left(\|x\| \frac{x}{\|x\|}\right)\right\|=\left\|S\left(\left(m \nu_{0}+\tau\right) \frac{x}{\|x\|}\right)\right\| \\
& \leq\left\|S\left(m \nu_{0} \frac{x}{\|x\|}\right)\right\| S\left(\tau \frac{x}{\|x\|}\right)\|\leq\| S\left(\nu_{0} \frac{x}{\|x\|}\right)\left\|^{m}\right\| S\left(\tau \frac{x}{\|x\|}\right) \| \\
& \leq M^{1+m}=M M
\end{aligned}
$$

( $\sigma=\nu_{0}^{-1} \log M \geq 0$ ) and the first part of the theorem is proved. The second part follows immediately.

3. Generator, infinitesimal generators and resolvents. It is convenient for our purpose to see a cone of infinitesimal generators associated with $S$ as an operator in $X^{+} \times Y$ into $Y$. We designate such an operator "generator". Thus the generator is $S$ is defined by

$$
A(x, y)=\lim _{t \downarrow 0} \frac{S(t x) y-y}{t}, \quad(x, y) \in X^{+} \times Y,
$$


and the domain $D(A)$ of $A$ is the subset of $X^{+} \times Y$ on which the limit exists. We establish some properties of the generator $A$ and the resolvents $R(\lambda ; A(x))$ of the $A(x)$ 's, $\lambda$ being in the resolvent set $\rho(A(x))$ of $A(x)$.

Theorem 2. Let $S$ be $C_{0}$ with $A$ its generator. Then

(a) $A$ is linear in the second argument and positive linear in the first;

(b) $A$ is closed.

Proof. For any fixed $x \in X^{+},\{S(t x): t>0\}$ is a one-parameter $C_{0}$ semigroup in $Y$, and $A(x)=A(x, \cdot)$ is its infinitesimal generator. The first part of (a) is therefore immediate and the second part follows as a consequence of [3, Theorem 10.8.5]. Consider (b). We adapt the classical technique for the infinitesimal generator. Let $\left(x_{n}, y_{n}\right)$ in $D(A)$ be such that $\left(x_{n}, y_{n}\right) \rightarrow$ $(x, y)$ in $X^{+} \times Y$ and $A\left(x_{n}, y_{n}\right) \rightarrow z$ in $Y$. Now $\int_{0}^{\alpha} S\left(t x_{n}\right) A\left(x_{n}\right) y_{n} d t=$ $\left[S\left(\alpha x_{n}\right)-I\right]_{y_{n}}$. The boundedness of a convergent sequence, Theorem 1 , the strong continuity of $S(x)$ and a $2-\epsilon$ argument show that $\lim _{n \rightarrow \infty} S\left(t x_{n}\right) A\left(x_{n}\right) y_{n}$ $=S(t x) z$ and $\left\|S\left(t x_{n}\right) A\left(x_{n}\right) y_{n}\right\| \leq K e^{t \sigma}$ for some $K$ and $\sigma$ positive and all $t>0$. Hence by the dominated convergence theorem and for similar reasons as above

$\lim _{n \rightarrow \infty} \int_{0}^{a} S\left(t x_{n}\right) A\left(x_{n}\right) y_{n} d t=\int_{0}^{a} S(t x) z d t=\lim _{n \rightarrow \infty}\left[S\left(\alpha x_{n}\right)-I\right] y_{n}=[S(\alpha x)-I] y$.

And, from the $C_{0}$ property of $S$,

$$
z=\lim _{a \downarrow 0} \frac{1}{\alpha} \int_{0}^{a} S(t x) z d t=\lim _{a ! 0} \frac{1}{\alpha}[S(\alpha x)-I] y .
$$

Thus $(x, y) \in D(A)$ and $A(x, y)=A(x) y=z$. This completes the proof.

Theorem 3. Let $S$ be $C_{0}$. Then

(1) for $x, x^{\prime} \in X^{+}$and $\lambda>\sigma_{x x^{\prime}}=\max \left(\sigma_{x}, \sigma_{x^{\prime}}\right)$,

$$
R(\lambda ; A(x)) R\left(\lambda ; A\left(x^{\prime}\right)\right)=R\left(\lambda ; A\left(x^{\prime}\right)\right) R(\lambda, A(x)) ;
$$

(2) if $B \subset X^{+}$is bounded, then there exist positive constants $M$ and $\sigma_{B}$ such that

$$
\left\|[R(\lambda ; A(x))]^{n}\right\| \leq M\left(\lambda-\sigma_{B}\right)^{-n} \quad \forall x \in B, \lambda>\sigma_{B}, n=1,2, \cdots .
$$

Proof. (1) follows from the integral representation of $R(\lambda ; A(x))$, the commutativity of the $S(x)$ 's and the boundedness of these linear operators. (2) similarly follows.

4. Generation. Finally, we consider the problem: What property should an operator $A$ possess to be the generator for $S$ ? 
Theorem 4. An operator $A$ in $X^{+} \times Y$ into $Y$ is the generator of a unique $C_{0}$ semigroup $S$ iff

(1) $A$ is additive in the first argument, linear in the second and closed;

(2) for each $x \in X^{+}, D(A(x, \cdot))$ is dense in $Y$;

(3) for any bounded set $B \subset X^{+}$there exist positive constants $M_{B}$ and $\sigma_{B}$ such that

$$
\left\|[R(\lambda ; A(x))]^{n}\right\| \leq M_{B}\left(\lambda-\sigma_{B}\right)^{-n} \quad \forall x \in B, \lambda>\sigma_{B}, y \in Y ;
$$

(4) for any $x, x^{\prime} \in X^{+}$and any $\lambda \in \rho(A(x)) \cap \rho\left(A\left(x^{\prime}\right)\right)$,

$$
R(\lambda ; A(x)) R\left(\lambda ; A\left(x^{\prime}\right)\right)=R\left(\lambda ; A\left(x^{\prime}\right)\right) R(\lambda ; A(x)) .
$$

Furthermore,

$$
S(x) y=\lim _{\lambda \rightarrow \infty} \exp (-\lambda) \sum_{n=0}^{\infty} \frac{\lambda^{n}[\lambda R(\lambda ; A(x))]^{n}}{n !} y
$$

for each $y \in Y$. and $x \in X^{+}$.

Proof. The "only if" part is clear. Consider the "if" part. $A$ is closed in $X^{+} \times Y$ and linear in the second argument. Hence $A(x, \cdot)=A(x)$ is a closed linear operator in $Y$. Along with hypotheses (2) and (3) it is well known [3, pp. 360-362] that for $x \in X^{+}, y \in Y$ and $\lambda>\sigma_{x}$

$$
S_{\lambda}(t x) y \stackrel{\text { def }}{=} S_{\lambda}(t ; A(x)) y \stackrel{\text { def }}{=} \exp (-\lambda t) \sum_{n=0}^{\infty} \frac{(\lambda t)^{n}[\lambda R(\lambda ; A(x))]^{n}}{n !} y
$$

converges uniformly for $t$ in any bounded subset of $[0, \infty)$. Hypothesis (3) makes the limit

$$
S(t x) y \stackrel{\text { def }}{=} S(t ; A(x)) y \stackrel{\text { def }}{=} \lim _{\lambda \rightarrow \infty} S_{\lambda}(t x) y
$$

converge uniformly for $t$ in any bounded subset of $[0, \infty)$ and $x$ in $B$. Then by a $3-\epsilon$ argument, $\lim _{t \downarrow 0} S(t x) y=y$ uniformly w.r.t. $x$ in B. And, therefore,

$$
\lim _{\|x\| \rightarrow 0} S(x) y=\lim _{\|x\| \rightarrow 0} S\left(\|x\| \frac{x}{\|x\|}\right) y=y \quad(y \in Y) .
$$

We show next that $S(x)$ possesses the semigroup property in $X^{+}$. Let $x, x^{\prime} \in X^{+}$be fixed and let $z$ be any one of $x, x^{\prime}$ and $x+x^{\prime}$.

(a) Then hypothesis (3) and the series representation of $S_{\lambda}(t x)$ give $\left\|S_{\lambda}(t z)\right\| \leq M e^{t \sigma}$ for some $\sigma>0$ and all $t>0, \lambda>\sigma$. 
(b) Since by hypothesis (4) the resolvents commute, then $R(\lambda ; A(z))$ commutes with $S_{\lambda}(t z) \forall \lambda>\sigma$ and $t \geq 0$.

(c) $d S_{\lambda}(t z) y / d t=S_{\lambda}(t z) \lambda A(z) R(\lambda ; A(z)) y \forall \lambda>\sigma, t>0$ and $y \in Y$.

(d) $\lim _{\lambda \rightarrow \infty} \lambda R(\lambda ; A(z)) y=y$.

(See, for instance, [8] for (c) and (d).) It follows that for $\lambda>\sigma$ and $y \epsilon$ $D(A(x)) \cap D\left(A\left(x^{\prime}\right)\right) \cap D\left(A\left(x+x^{\prime}\right)\right) \stackrel{\text { def }}{=} D$,

$$
\begin{aligned}
&\left\|S_{\lambda}\left(x+x^{\prime}\right) y-S_{\lambda}(x) S_{\lambda}\left(x^{\prime}\right) y\right\| \\
&=\left\|\int_{0}^{1} \frac{d}{d t}\left(S_{\lambda}\left((1-t)\left(x+x^{\prime}\right)\right) S_{\lambda}(t x) S_{\lambda}\left(t x^{\prime}\right) y\right) d t\right\| \\
&= \| \int_{0}^{1} S_{\lambda}\left((1-t)\left(x+x^{\prime}\right)\right) S_{\lambda}(t x) S_{\lambda}\left(t x^{\prime}\right) \\
& \cdot\left[\lambda A(x) R(\lambda ; A(x))+\lambda A\left(x^{\prime}\right) R\left(\lambda ; A\left(x^{\prime}\right)\right)-\lambda A\left(x+x^{\prime}\right) R\left(\lambda ; A\left(x+x^{\prime}\right)\right)\right] y d t \| \\
& \leq \int_{0}^{1} M^{3} e^{\sigma(1-t)} e^{2 t \sigma} \|\left[\lambda R(\lambda ; A(x)) A(x)+\lambda R\left(\lambda ; A\left(x^{\prime}\right)\right) A\left(x^{\prime}\right)\right. \\
&= \sigma^{-1} M^{3} e^{\sigma}\left(e^{\sigma}-1\right) \|\left[\lambda R(\lambda ; A(x)) A(x)-A(x)+\lambda R\left(\lambda ; A\left(x^{\prime}\right)\right) A\left(x^{\prime}\right)\right. \\
&\left.\quad-A\left(x^{\prime}\right)+A\left(x+x^{\prime}\right)-\lambda R\left(\lambda ; A\left(x+x^{\prime}\right)\right) A\left(x+x^{\prime}\right)\right] y \|
\end{aligned}
$$

as $\lambda \rightarrow \infty$ for all $y \in D$; the last equality holding because $A$ is additive in the first argument (hypothesis (1)). It is a consequence of hypothesis (2) that $D$ is dense in $Y$. Since (a) also holds, it follows by the Banach-Steinhaus theorem that $\left\|S_{\lambda}\left(x+x^{\prime}\right) y-S_{\lambda}(x) S_{\lambda}\left(x^{\prime}\right) y\right\| \rightarrow 0$ as $\lambda \rightarrow \infty$ for all $y \in$ $Y$. Hence $S\left(x+x^{\prime}\right) y=S(x) S\left(x^{\prime}\right) y$ for all $x, x^{\prime} \in X^{+}, y \in Y$. Thus $\left\{S(x): x \in X^{+}\right\}$is a $C_{0}$ semigroup in $Y$.

The rest of the proof is routine.

Theorem 5. An operator $A$ in $X^{+} \times Y$ into $Y$ is the generator of a partially $C_{0}$ semigroup $S$ iff $A$ is additive in the first argument; for each $x \in X^{+}, A(x)$ is the infinitesimal generator of a $C_{0}$ semigroup on $Y$; and the resolvents of the $A(x)$ 's commute.

Proof. Similarly as above. 


\section{REFERENCES}

1. V. A. Babalola, Semigroups of operators on locally convex spaces, Trans. Amer. Math. Soc. 199 (1974), 163-179.

2. J. Chabrowski and G. Kubczonok, Sur les semi-groupes à n paramètres des opérateurs linéaires, Studia Math. 33 (1969), 13-18. MR 39 \#7463.

3. E. Hille and R. S. Phillips, Functional analysis and semi-groups, rev. ed., Amer. Math. Soc. Colloq. Publ., vol. 31, Amer. Math. Soc., Providence, R. I., 1957. MR 19, 664 .

4. O. A. Ivanova, Certain theorems on an n-parametric semigroup of bounded linear operators and their application in the theory of functions, Teor. Funkcii Funkcional. Anal. i Priložen. Vyp. 2 (1966), 35-41. (Russian) MR 33 \#7870.

5. R. P. Langlands, On Lie semi-groups, Canad. J. Math. 12 (1960), 686-693. MR $22 \# 12401$.

6: - Some holomorphic semi-groups, Proc. Nat. Acad. Sci. U.S.A. 46 (1960), 361-363. MR 31 \#1566.

7. I. Miyadera, Semi-groups of operators in Fréchet space and applications to partial differential equations, Tôhoku Math. J. (2) 11 (1959), 162-183. MR 21 \#7445.

8. C. I. Tulcea, Suboperative functions and semi-groups of operators, Ark. Mat. 4 ( 1960), 55-61. MR $26 \# 4194$.

DEPARTMENT OF MATHEMATICS, UNIVERSITY OF IBADAN, IBADAN, NIGERIA (Current address)

SCHOOL OF MATHEMATICS, THE INSTITUTE FOR ADVANCED STUDY, PRINCETON, NEW JERSEY 08540 